\title{
Prenatal Diagnosis of Osteopathia Striata with Cranial Sclerosis in a Male Fetus with a $\sim 330 k b$ Deletion of Xq11.1 Involving the WTX Gene
}

\author{
Patrick L. Wilson ${ }^{1, *}$, Ashley Davis ${ }^{2}$, Jean Ricci Goodman ${ }^{1,4}$, Lauren Notley ${ }^{1}$, Shibo Li $^{2}$, Ji-Yun Lee ${ }^{2,5}$, Zhongxin \\ $\mathbf{Y u}^{3}$, Klaas J. Wierenga ${ }^{2}$, Andrew F. Wagner ${ }^{1}$ \\ ${ }^{1}$ Department of Obstetrics and Gynecology, Maternal/Fetal Medicine, University of Oklahoma Health Sciences Center, Oklahoma City, \\ USA \\ ${ }^{2}$ Department of Pediatrics, Genetics Section, University of Oklahoma Health Sciences Center, Oklahoma City, USA \\ ${ }^{3}$ Department of Pathology, University of Oklahoma Health Sciences Center, Oklahoma City, USA \\ ${ }^{4}$ Deparment of Obstetrics and Gynecology, Maternal/Fetal Medicine, Loyola University, Stritch School of Medicine, Chicago, USA \\ ${ }^{5}$ Department of Pathology, Korea University, Seoul, Korea \\ *Corresponding author: Patrick-L-Wilson@ouhsc.edu
}

Received December 29, 2012; Revised February 15, 2013; Accepted February 16, 2013

\begin{abstract}
Osteopathia striata with cranial sclerosis (OSCS) is a rare X-linked dominant genetic disorder resulting mutation in the WTX gene. Clinically, OSCS presents with linear striations in the metaphyseal region of the long bones and pelvis in combination with sclerosis of the cranium and face. A twenty-seven year old G5T1P3A0L2 woman with a history of peri- and neonatal infant male deaths was referred to us at 22 weeks, 6 days into her recent pregnancy. Ultrasound evaluation identified a male fetus, bilaterally enlarged lateral ventricles, a cloverleaf skull, suspected bilateral cleft lip, nuchal thickening, bilateral bowed radii and ulnae, gastroschisis with herniated viscera, bilateral absent fibula and clubfeet. The results of prenatal testing identified a male fetus with a 330kb Xq11.1 deletion involving the entire WTX gene. Initial microarray analysis of maternal blood identified a normal female karyotype, 46,XX. FISH analysis with a BAC clone mapped to the WTX gene identified low-level mosaicism in blood and buccal samples, $17 \%$ and $15 \%$, respectively, which explained the negative maternal microarray result.
\end{abstract}

Keywords: Osteopathia striata with cranial sclerosis (OSCS), Wilms tumor on the X-chromosome (WTX) gene, Microarray, Mosaic, Osteopetrosis, Germline mosaicism

\section{Introduction}

Osteopetrosis, or "marble bone disease," refers to a group of rare heritable, skeletal disorders. Phenotypically, there is an increase in bone density on radiographs which is due to a failure of osteoclasts to differentiate or function properly [1].

A subtype of osteopetrosis is osteopathia striata with cranial sclerosis (OSCS), which is a rare genetic disorder that is most often seen with X-linked inheritance patterns. Clinically, OSCS presents with longitudinal striations of osteosclerosis in the long bones. More importantly, OSCS presents with osteosclerosis in the bones of the cranium and face. This type of osteosclerosis leads to disfigurement and increased pressure on the cranial nerves, which leads to disabilities, e.g. deafness. OSCS has phenotypic variability in females and male. In females, OSCS features are macrocephaly, cleft palate, mild learning disabilities, sclerosis of the long bones and skull and longitudinal striations. For males, OSCS causes fetal or neonatal death. Males that do survive have the features of females with OSCS along with hyperostosis, cardiac, intestinal and genitourinary malformations [2,3]. Other features of OSCS may include: clubfoot; small ventricular septal defects, aortic stenosis; clinodactyly; dental and visual problems; hydrocephalus; hypertelorism, flat nasal bridge; scoliosis of the back; long fingers, facial nerve palsy; and mild mental retardation.

The gene responsible for OSCS has been mapped to the locus Xq11.1. The phenotype is thought to be due to a mutation in the WTX gene. WTX, also referred to as Wilms Tumor Gene on the $\mathrm{X}$ Chromosome, is a $7.5 \mathrm{~Kb}$ transcript that encodes for a protein that consists of 1,135 amino acids. If the WTX gene is mutated, WNT signaling will increase. Therefore, WTX normally acts as a repressor of canonical WNT signaling [4].

Here, we report a case of osteopathia striata with cranial sclerosis in a male baby. The mother had two previous pregnancies in which the male fetus had multiple anomalies.

\section{Case Presentation}

The patient originally presented to us during her first pregnancy in 2002 at 27-6/7 weeks gestation because of anhydramnios and abnormal outside ultrasound. The ultrasound identified a male fetus with, frontal bossing, mild hydrocephalus, short limbs, indeterminate hands and feet, a small chest, and kidneys that were dilated and echogenic. At 29 weeks, the patient was diagnosed with eclampsia and delivered her son via cesarean section. 
Postmortem physical examination revealed the presence of micrognathia, low set posteriorly rotated ears, cleft tongue, a low hairline, hypertelorism, flattened nasal bridge, short limbs (absent fibulae per roentgenogram), bilateral first and second finger syndactyly, single palmar crease, and third degree hypospadias (Figure $1 \mathrm{~A}$ ). The lungs were moderately hypoplastic and showed signs of neonatal respiratory distress syndrome. The heart contained a patent ductus arteriosus and a $0.2 \mathrm{~cm}$ diameter ventricular septal defect. Evaluation of the kidneys showed extensive replacement of parenchyma by perilobar nephroblastomatosis and a nephroblastoma. The left kidney contained an internal cyst. Karyotype analysis was 46,XY.

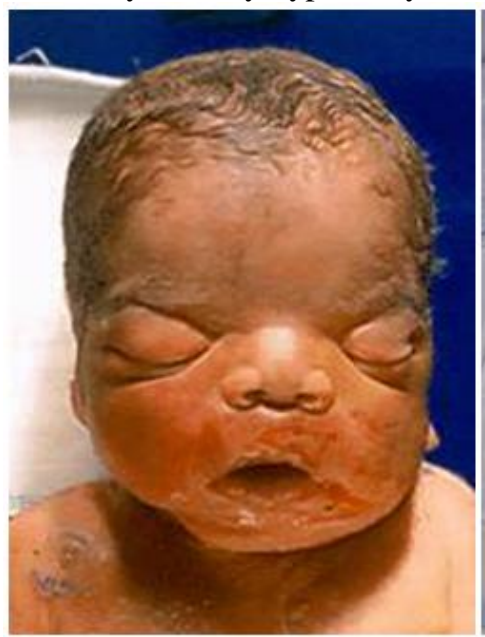

A

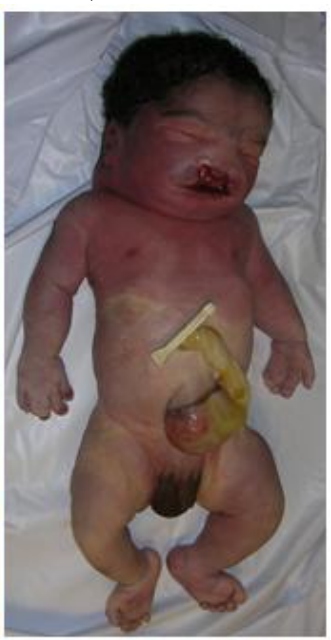

B
Figure 1. Autopsy Photos: A. 2002 Pregnancy, Face with prominent forehead, hypertelorism and micrognathia. B. 2009 Pregnancy, Anterior view with omphalocele.

In 2003, the patient delivered a healthy girl at 31 weeks via repeat cesarean section due to preeclampsia.

In 2005, the patient was pregnant once again with a male fetus. Ultrasound at 23-4/7 weeks identified a Dandy Walker malformation, limb shortening with wishbone shaped legs, and an omphalocele. During the $27^{\text {th }}$ week of the pregnancy, the patient experienced a placental abruption and delivered a stillborn son via repeat cesarean section. Postnatal karyotype analysis was consistent with prenatal karyotype: 46,XY.

In 2006 , the patient was pregnant with a female fetus. She presented to us at $16-2 / 7$ and $25-0 / 7$ weeks into the pregnancy. Ultrasound evaluation at each visit identified a normally developing fetus. Amniocentesis performed at the 16-week visit returned a karyotype of 46,XX. A healthy baby girl was delivered via repeat cesarean section during at $38-0 / 7$ weeks.

In 2009 , the patient was referred for an ultrasound at 20-6/7 weeks into the pregnancy. The procedure identified a phenotypical male fetus with bilaterally enlarged lateral ventricles, a cloverleaf skull, suspected bilateral cleft lip, nuchal thickening, bilateral bowed radii and ulnae, gastroschisis with herniated viscera, bilateral absent fibula and clubfeet. Oligohydramnios was present with an amniotic fluid index of $4.5 \mathrm{~cm}$. At $38-0 / 7$ weeks, the patient delivered via repeat low transverse cesarean section (Figure $1 \mathrm{~B}$ ). The baby was born alive with APGARs of 8 at 1 minute, 1 at 5 minutes, and 1 at 10 minutes. NICU physicians administered epinephrine; however, resuscitation was unsuccessful.

Due to the patient's history of male fetuses with multiple abnormalities, an extensive cytogenetic evaluation was performed to determine the cause of the abnormalities and possible recurrence risk in future pregnancies. During the 2009 pregnancy, an amniocentesis was performed during the initial 20-week visit. A normal male karyotype of 46,XY was returned. Prenatal CGH-microarray analysis identified an approximately $330 \mathrm{~kb}$ deletion on the X-chromosome at position Xq11.1 involving the WTX gene (Figure 2). Karyotype and microarray analysis on a maternal blood sample were both normal.

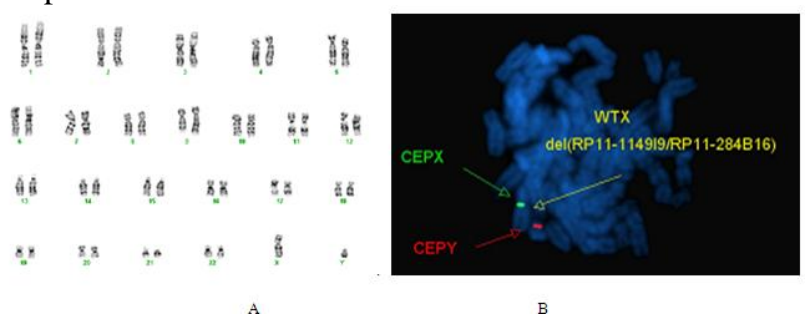

Figure 2. Representative Cytogenetic Results from Patient's Sons: A. Karyotype: 46, XY. B. FISH showing deletion of WTX gene.

The maternal finding caused us to wonder if she were possibly mosaic for the WTX deletion due to the presence of similarly affected sons by different partners. Permission was obtained to re-evaluate stored samples from the previous pregnancies. FISH analysis with a BAC clone mapped to the WTX gene was performed on stored slides from the amniocenteses that were performed during the 2002 and 2005 pregnancies. Each was positive for the WTX gene deletion. Follow-up FISH analysis of the maternal blood sample from the 2009 pregnancy identified low level mosaicism, 17 of 100 cells, which microarray analysis was not able detect. A buccal swab sample showed 15 of 100 cells to also be mosaic for the gene deletion (Figure 3).
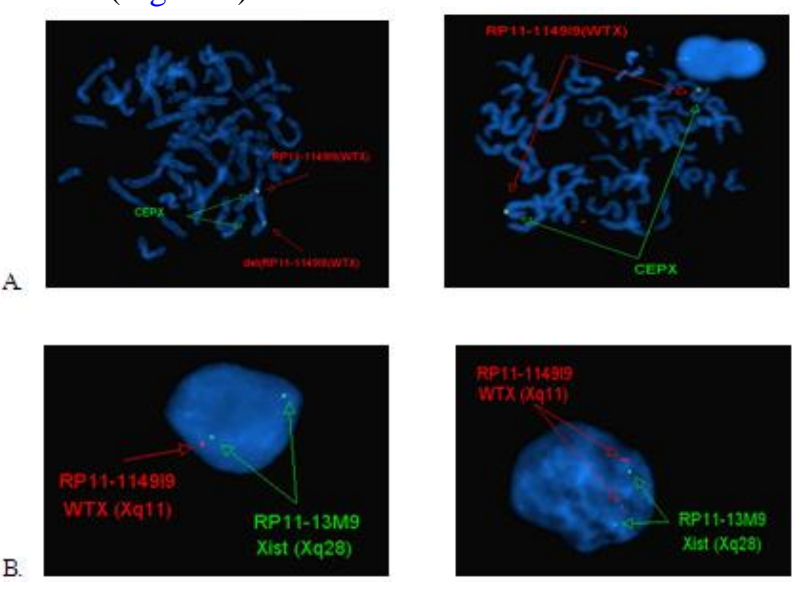

Figure 3. Maternal FISH Results. Identification of low level mosaicism for an $\sim 330 \mathrm{~kb}$ deletion at Xq11.1(63,314,999-63,614,999) involving the WTX, ASB12, and MTMR8 genes. (NimbleGen 135K, Roche NimbleGen, Inc., UCSC hg18 human genome: NCBI build 36, Mar 2006, RefSeq, and GenBank databases)

A. Maternal blood lymphocytes, 17 of 100 cells, and B. Maternal buccal swab showing low level mosaicism, 15 of 100 cells.

\section{Discussion}


Osteopathia striata with cranial sclerosis (OSCS) is an $\mathrm{X}$-linked dominant condition resulting from nonsense mutations and whole gene deletions of the Wilms tumor on the X-chromosome (WTX) gene [1], also known as FAM123B. In females, the condition is generally benign. The female phenotype consists of long bone striations, cranial sclerosis, cranial dysmorphisms (frontal bossing, macrocephaly, wide nasal bridge, hypertelorism, low set ears), clefted or highly arched palate with dental anomalies, conductive or sensorineural hearing loss, developmental delay, and mental retardation. The hemizygous male phenotype is often more severe than what is seen in females. It consists of significant skeletal sclerosis, absent metaphyseal striations, macrocephaly, facial dysmorphisms, micrognathia, prominent lumbar lordosis, joint luxaation, camptodactyly, flexion contractures, omphalocele, duodenal web, gut malrotation, inguinal hernia, Hirschsprung disease, patent ductus arteriosus, atrial and ventricular septal defects, left ventricular non-compaction, tricuspid insufficiency, and multicystic kidneys [2]. Within and among families there can be a variable range of penetrance and phenotypic expression regardless of the WTX mutation.

The WNT family of proteins consists of secreted signaling molecules that regulate cell-to-cell interactions during embryogenesis. As a repressor of WNT signaling, WTX helps mediate the repressive effects of the ossification of bones that develop from a cartilage scaffold (long bones) and that differentiate from messenchymal precursors (calvaria). WNT-related signaling defects have been attributed to osteoblast activation, inhibition of osteoclast differentiation, or re-direction of pluripotent stem cell differentiation from an adipocyte to chondrocyte to an osteoblast depending on the level within the pathway and/or developmental timing of activation of canonical WNT signaling [1]. The absence of WTX during this period of development contributes to bone overgrowth by increasing the commitment of messenchymal progenitor cells towards osteoblastogenesis [3].

WTX is a member of a gene family, FAM123, comprised of three genes with no significant homology to known functional domains (3). WTX consists of two exons that are transcribed into a $\sim 7.5 \mathrm{~kb}$ mRNA transcript that encodes a 1,135 amino acid protein. Two isoforms of the protein, resulting from alternative splice donor and acceptor sites within exon 2 , are present within cells. The full-length isoform, $\mathrm{WTX}_{\mathrm{s} 1}$, resides in the plasma membrane and cytoplasm. $\mathrm{WTX}_{\mathrm{s} 1}$ possess three adenomatous polyposis coli (APC) binding sites that facilitate its interaction with APC at the plasma membrane. The short isoform, $\mathrm{WTX}_{\mathrm{s} 2}$, lacks amino acid residues 50326 in the N-terminal portion of the protein. WTX $_{\mathrm{s} 2}$ resides in the nucleus where it forms a complex with $\beta$ catenin and the destruction complex (AXIN1, $\beta$-transducin repeat-containing protein2, and APC). It also promotes the ubiquitylation and degradation of $\beta$-catenin. Both WTX isoforms are able to bind $\beta$-catenin $[2,4,5,6]$.

Formerly OSCS was thought to be universally lethal in males. Males with WTX mutations that produced a WTXs1 isoform lacking an intact phospholipids binding (PtdIns $(4,5) \mathrm{P}_{2}$ ) and/or APCD1 resulted in death. However, recent studies have shown that this is not always the case. Three males, two of whom survived into adulthood, with mutations in the 5' region of WTX had a mild phenotype calling into question the relationship between the location of the WTX mutation and its resulting phenotype [2,5]. Non-mosaic males that possess a WTX mutation $3^{\prime}$ to the acidic domain and those who are mosaic for a WTX deletion have a milder phenotype [7].

The present case is an excellent representation of the progression of genetic testing. When the patient originally presented the gene responsible for OSCS had not been identified, and its deletion could not be visualized by standard karyotype. Years later, with the introduction of microarray analysis, a deletion on the X-chromosome encompassing the entire WTX gene was identified. Around the time of the 2009 pregnancy, the WTX gene was determined to be causative for OSCS. This finding allowed us to properly diagnosis the cause of the lethal changes in the patient's sons. However, because microarray analysis does not identify low level mosaicism, FISH analysis with a BAC clone mapped to the WTX gene was performed on blood and buccal cells from the patient, $17 \%$ and $15 \%$ respectively.

The final question to be answered is the status of the patient's two pre-teen daughters. The presence of low level somatic mosaicism raises the issue that the patient might also be a gonadal mosaic. Such mosaicism may result from one of two events: (1) in early embryogenesis a mutation occurs in a germ cell prior to meiosis and is carried in that cell's daughter cells or (2) a mutation occurs in a somatic cell prior to its separation into germ cells and is present in both somatic and germ cells. The timing of the mutation will ultimately determine the percentage of germ cells that carry the mutation, ranging from a few to $50 \%[8,9]$. Using the Van der Meulen et al. model for the recurrence risk for gonadal mosaics, the recurrence risk for this patient with three affected sons and two untyped unaffected daughters is $36.6 \%$ [10]. If the carrier status of her daughters is determined, this figure can be adjusted accordingly. This information may prove useful to the patient should she choose to have more children in the future.

In conclusion, this patient and her family represent an interesting case study in the diagnosis of OSCS. The use of various molecular and cytogenetic techniques allowed for the diagnosis of the condition in her affected sons and her low level mosaicism. To our knowledge, it also represents the second case of documented WTX mosaicism and the first in a female [2].

\section{References}

[1] Jenkins, Z.A., van Kogelenberg, M., Morgan, T., Jeffs, A., Fukuzawa, R., Pearl, E., Thaller, C., Hing, A.V., Porteous, M.E., Garcia-Miñaur, S., Bohring, A., Lacombe, D., Stewart, F., Fiskerstrand, T., Bindoff, L., Berland, S., Adès, L.C., Tchan, M., David, A., Wilson, L.C., Hennekam, R.C., Donnai, D., Mansour, S., Cormier-Daire, V., and Robertson, S.P, "Germline mutations in WTX cause a sclerosing skeletal dysplasia but do not predispose to tumorigenesis," Nature Genetics, 41 (1). 95-100. Jan. 2009.

[2] Holman, S.K., Daniel, P., Jenkins, Z.A., Herron, R.L., Morgan, T, Savarirayan, R., Chow, C.W,, Bohring, A., Mosel, A., Lacombe, D., Steiner, B., Schmitt-Mechelke, T., Schroter, B., RaasRothschild, A., Miñaur, S.G., Porteous, M., Parker, M., Quarrell, O., Tapon, D., Cormier-Daire, V., Mansour, S., Nash R, Bindoff LA, Fiskerstrand T, and Robertson, S, "The male phenotype in osteopathia striata congenita with cranial sclerosis,"American Journal of Medical Genetics A, 155A (10). 2397-408. Oct. 2011.

[3] Moisan, A., Rivera, M.N., Lotinun, S., Akhavanfard, S., Coffman, E.J., Cook, E.B., Stoykova, S., Mukherjee, S., Schoonmaker, J.A., Burger, A., Kim, W.J., Kronenberg, H.M., Baron, R., Haber, D.A., 
and Bardeesy, N, "The WTX tumor suppressor regulates mesenchymal progenitor cell fate specification," Developmental Cell, 20 (5). 583-96. May 2011.

[4] Perdu, B., de Freitas, F., Frints, S.G., Schouten, M., SchranderStumpel, C., Barbosa, M., Pinto-Basto, J., Reis-Lima, M., de Vernejoul, M.C., Becker, K., Freckmann, M.L., Keymolen, K., Haan, E., Savarirayan, R., Koenig, R., Zabel, B., Vanhoenacker, F.M., and Van Hul, W, "Osteopathia striata with cranial sclerosis due to WTX gene defect," Journal of Bone and Mineral Research, 25 (1). 82-90. Jan.2010.

[5] Perdu, B., Lakeman, P., Mortier, G., Koenig, R.., Lachmeijer, A., and Van Hul, W, "Two novel WTX mutations underscore the unpredictability of male survival in osteopathia striata with cranial sclerosis," Clinical Genetics, 80 (4). 383-8. Oct. 2011.

[6] Huff, V, "Wilms' tumours: about tumour suppressor genes, an oncogene, and chameleon gene," Nature Reviews, Cancer, 11 (2). 111-21. Feb. 2011.
[7] Chénier, S., Noor, A., Dupuis, L., Stravropoulos, D.J., MendozaLondona R. "Osteopathia striata with cranial sclerosis and developmental delay in a male with a mosaic deletion in chromosome region Xq11.2," American Journal of Medical Genetics, Part A, 158A (11). 2946-52. Nov. 2012.

[8] Horn, D., Delaunoy, J-P., and Kunze, J, "Prenatal diagnosis of Coffin-Lowry syndrome demonstrates germinal mosaicism confirmed by mutation analysis," Prenatal Diagnosis, 21 (10). 881-4. Oct. 2001

[9] Pilozzi-Edmonds, L., Maher, T., Basran, R., Milunsky, A., AlThihli, K., Braverman, N., and Alafares, A, "Fraternal twins with Aarskog-Scott syndrome due to maternal germline mosaicism," American Journal of Med Genetics Part A. 155A (8). 1987-90. Aug. 2011

[10] Van der Meulen, M.A., van der Meulen, M.J., and te Meermen, G.J, "Recurrence risk for germinal mosaics revisited," Journal of Medical Genetics, 32 (2). 102-4. Feb. 1995. 